\title{
Development of universal antidotes to control aptamer activity
}

\author{
Sabah Oney ${ }^{1-3,7}$, Ruby T S Lam ${ }^{4}$, Kristin M Bompiani ${ }^{1-3}$, Charlene M Blake ${ }^{1-3}$, George Quick ${ }^{1}$, Jeremy D Heidel ${ }^{5}$, \\ Joanna Yi-Ching Liu ${ }^{5}$, Brendan C Mack ${ }^{6}$, Mark E Davis ${ }^{6}$, Kam W Leong $^{4}$ \& Bruce A Sullenger ${ }^{1-3}$
}

\begin{abstract}
With an ever increasing number of people taking numerous medications, the need to safely administer drugs and limit unintended side effects has never been greater. Antidote control remains the most direct means to counteract acute side effects of drugs, but, unfortunately, it has been challenging and cost prohibitive to generate antidotes for most therapeutic agents. Here we describe the development of a set of antidote molecules that are capable of counteracting the effects of an entire class of therapeutic agents based upon aptamers. These universal antidotes exploit the fact that, when systemically administered, aptamers are the only free extracellular oligonucleotides found in circulation. We show that proteinand polymer-based molecules that capture oligonucleotides can reverse the activity of several aptamers in vitro and counteract aptamer activity in vivo. The availability of universal antidotes to control the activity of any aptamer suggests that aptamers may be a particularly safe class of therapeutics.
\end{abstract}

Oligonucleotide therapeutics (that is, small interfering RNAs (siRNAs) and aptamers) have gained much attention as potential treatments for a variety of diseases ${ }^{1-6}$. Recent scientific advancements have led to an exponential increase in the number of such molecules going through clinical trials. Aptamers are single-stranded nucleic acid molecules that bind and inhibit protein targets ${ }^{7}$. We were the first to show the potential therapeutic utility of aptamers by inhibiting HIV-replication with a TAR aptamer ${ }^{8}$ and others continue to develop this clinically ${ }^{9}$. Building on this principle and using combinatorial libraries of nucleic acids and the systematic evolution of ligands by exponential enrichment (SELEX) method ${ }^{10,11}$, many aptamers have been developed that have potential therapeutic value ${ }^{7}$.

A number of aptamers targeting coagulation factors have been described that inhibit clot formation and represent potential anticoagulant therapeutics ${ }^{7,12,13}$. Regrettably, anticoagulants engender complications, such as considerable bleeding, that increase patient morbidity and mortality ${ }^{14}$. Currently, only one anticoagulant and antidote pair, heparin and protamine, is routinely used in clinics ${ }^{15,16}$. Antidote control provides the safest means to regulate drug action and minimize side effects. Unfortunately, it has been challenging to develop antidotes for antibody and small-molecule drugs, owing to the inability to distinguish therapeutic antibodies or small-molecule drugs from their endogenous counterparts in a patient's body.

Previously, we described how customized antidote oligonucleotides could be created to specifically reverse the activity of particular aptamers targeting coagulation factor IXa (FIXa) and von Willebrand factor (vWF) by exploiting Watson-Crick base pairing to generate duplex RNAs ${ }^{1,16-18}$. This approach yielded rationally designed drug-antidote pairs but has several limitations ${ }^{15,16,19}$. First, it is cost prohibitive to develop such customized and expensive oligonucleotide antidotes for every therapeutic aptamer. In addition, we have observed that it is challenging to develop such antidotes to highly structured aptamers. Finally, because the resulting aptamer-antidote complex is a double stranded RNA, the complex may activate the innate immune response, leading to inflammation ${ }^{20}$.

Here we describe an approach that overcomes these limitations in antidote design and yields universal antidote molecules that can control the activity of many aptamers, regardless of their sequence. We recognized that oligonucleotide-based drugs are different from antibody and small-molecule drugs in that no endogenous counterparts for oligonucleotide drugs are normally free in circulation. Thus, we hypothesized that molecules that can sequester oligonucleotides in a sequence-independent manner should be able to function as universal antidotes for extracellular oligonucleotide-based drugs in animals and humans.

\section{RESULTS}

Because two aptamers selected to different targets (thrombin and vascular endothelial growth factor) have been shown to interact with the heparin-binding domain on their target proteins $s^{5,21}$, we first investigated whether protamine, the antidote for heparin, could reverse the activity of two aptamers that target FIXa (aptamer 9.3t) and FXa (aptamer 11F7T). The activated partial thromboplastin time (APTT) assay is used for monitoring anticoagulation therapy, and we have previously shown that this assay can also be used to follow the anticoagulant effects of both FIXa and FXa aptamers ${ }^{16,19}$ (J. Layzer and B.A.S., unpublished data). These aptamers have markedly different primary sequences and secondary structures (as predicted by mFold,

${ }^{1}$ Duke Translational Research Institute, Duke University Medical Center, Durham, North Carolina, USA. ${ }^{2}$ University Program in Genetics and Genomics, Duke University, Durham, North Carolina, USA. ${ }^{3}$ Department of Surgery, Duke University Medical Center, Durham, North Carolina, USA. ${ }^{4}$ Department of Biomedical Engineering, Duke University, Durham, North Carolina, USA. ${ }^{5}$ Calando Pharmaceuticals, Pasadena, California, USA. ${ }^{6}$ Department of Chemical Engineering, California Institute of Technology, Pasadena, California, USA. ${ }^{7}$ Present address: b3bio, Inc., Research Triangle Park, North Carolina, USA. Correspondence should be addressed to B.A.S. (bruce.sullenger@duke.edu).

Received 28 July 2008; accepted 13 May 2009; published online 4 October 2009; doi:10.1038/nm.1990 
a

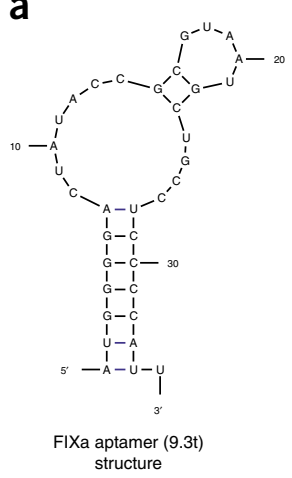
structure

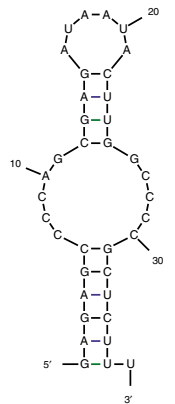

FXa aptamer (11F7T)

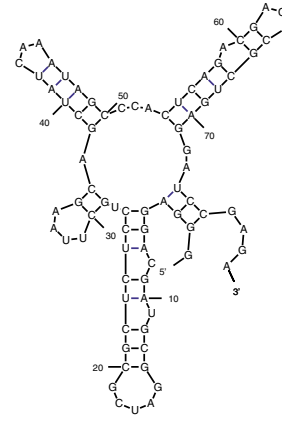

VWF aptamer (9.3)

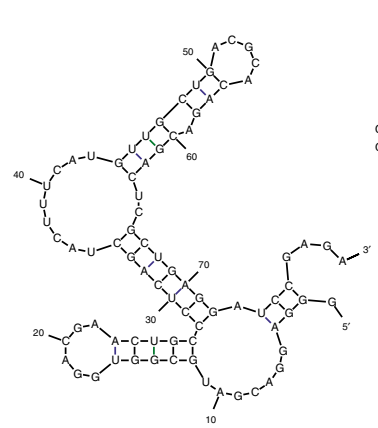

VWF aptamer (9.14) structure

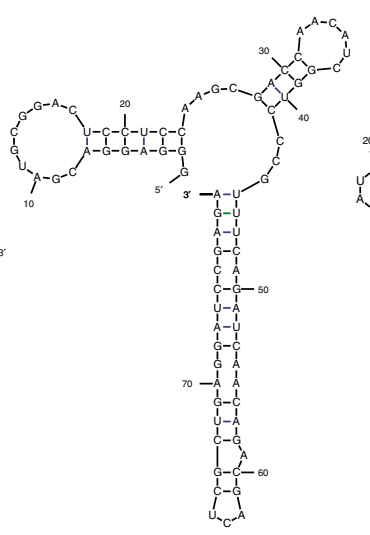

FVIla aptamer (7S-1) structure

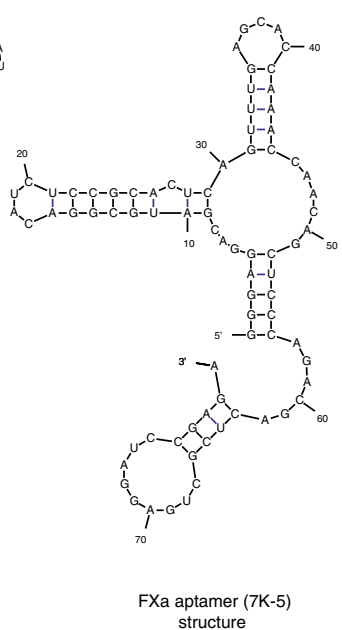

structure
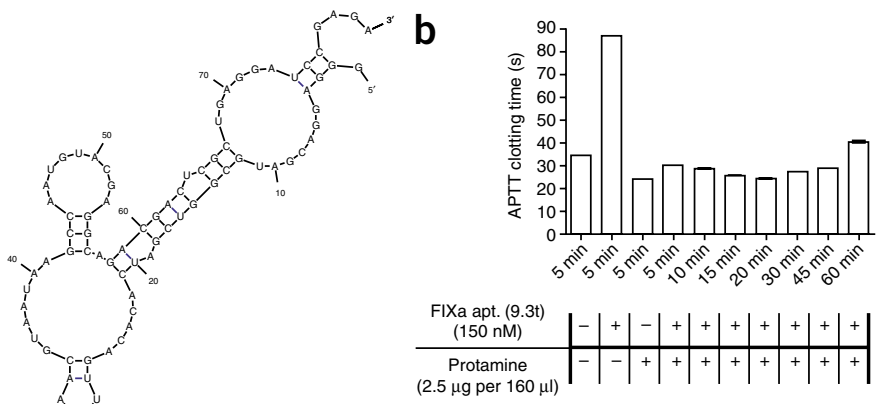

C
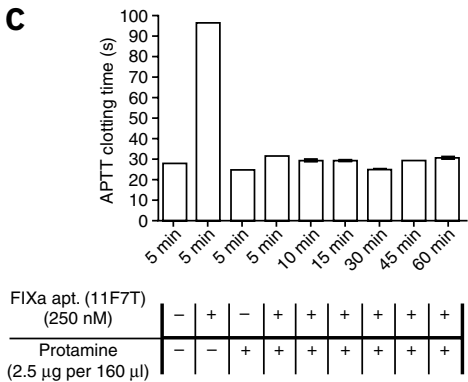

d
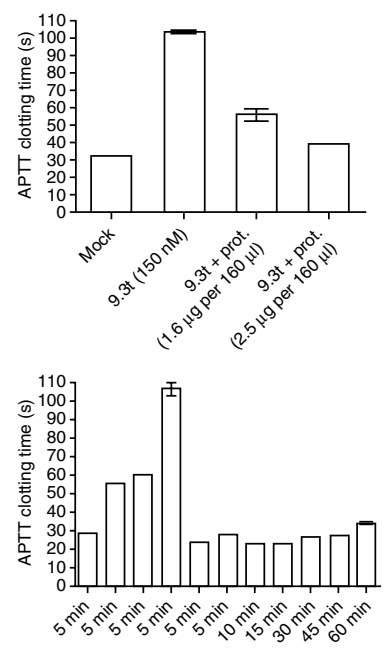

Figure 1 The structures of aptamers used in this study and protamine-mediated reversal of anticoagulant aptamer function. (a) The primary sequence and predicted secondary structures of FIXa (9.3t), FXa (11F7T), VWF (9.3), FII (R9D-14), VWF (9.14), FVII (7S-1), FX (7K-5) and FIX (9D-6) aptamers. (b) APTT clotting time of normal human plasma in the presence of aptamer $9.3 \mathrm{t}$ (150 nM), with and without the addition of protamine $(2.5 \mu \mathrm{g}$ per $160 \mu \mathrm{l}$ ). (c) APTT clotting time of normal human plasma in the presence of aptamer 11F7T (250 nM), with and without the addition of protamine $(2.5 \mu \mathrm{g}$ per $160 \mu \mathrm{l})$. (d) APTT clotting time of normal human plasma in the presence of aptamer $9.3 \mathrm{t}$ (150 nM) and increasing concentrations of protamine. (e) APTT clotting time of normal human plasma in the presence of aptamers 9.3t and 11F7T, with and without the addition of protamine. The data are plotted as the means \pm s.e.m. for three independent measurements.

structure $\mathbf{e}$

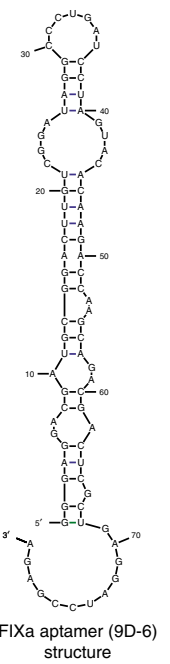

Fig. 1a) ${ }^{22}$ and target different proteins in the coagulation cascade. We found that aptamer $9.3 \mathrm{t}$ is a potent anticoagulant (Fig. 1b). However, addition of protamine neutralized the anticoagulant effects of this aptamer within $5 \mathrm{~min}$ (Fig. 1b). Similarly, aptamer 11F7T is a potent anticoagulant, and protamine also rapidly neutralized the activity of this aptamer (Fig. 1c). In both cases, protamine $(2.5 \mu \mathrm{g})$ was able to totally reverse the aptamers' activity in an APTT clotting assay (Fig. 1d) at a $50 \%$ lower concentration than is routinely used to reverse heparin's anticoagulant activity. Moreover, protamine was able to rapidly reverse the anticoagulant activity of the two aptamers simultaneously (Fig. 1e), and such reversal was maintained for at least $1 \mathrm{~h}$.

Although protamine is routinely used to reverse the activity of heparin after cardiopulmonary bypass surgery, protamine administration is associated with several side effects, including increased pulmonary artery pressure, decreased systolic and diastolic blood pressure, impaired myocardial oxygen consumption, and reduced cardiac output, heart rate and systemic vascular resistance ${ }^{15,23-26}$. Therefore, we sought to identify other agents that could rapidly reverse the activity of aptamers. We first screened a number of nucleic acid binding polymers for their ability to act as antidotes for aptamer 9.3t (Supplementary Table 1). Several of the polymers were able to completely reverse the activity of the aptamer within 5 min (Fig. 2a). To better understand why certain polymers were more effective than others, we measured the interactions of the different polymers with aptamer $9.3 \mathrm{t}$ by isothermal titration calorimetry (ITC). We used a two-site model to interpret the ITC data for the interactions between the cationic polymers and aptamer 9.3t. Binding constants and some thermodynamic parameters are summarized in Supplementary Table 2. As expected, all of the interactions were entropy driven, except for with polyamidoamine dendrimer, 1,4-diaminobutane core, G3 (PAMAM), and most were also enthalpy driven. Although no conclusions can be drawn about the mechanisms of the interactions, the trends of binding strength, in the two-site model, were consistent with the results from APTT screening. Protamine, $\beta$-cyclodextrin-containing polycation (CDP), $\beta$-cyclodextrin-containing polycation, imadazole-containing variant (CDP-Im), polyphosphoramidate polymer 


\section{TECHNICAL REPORTS}
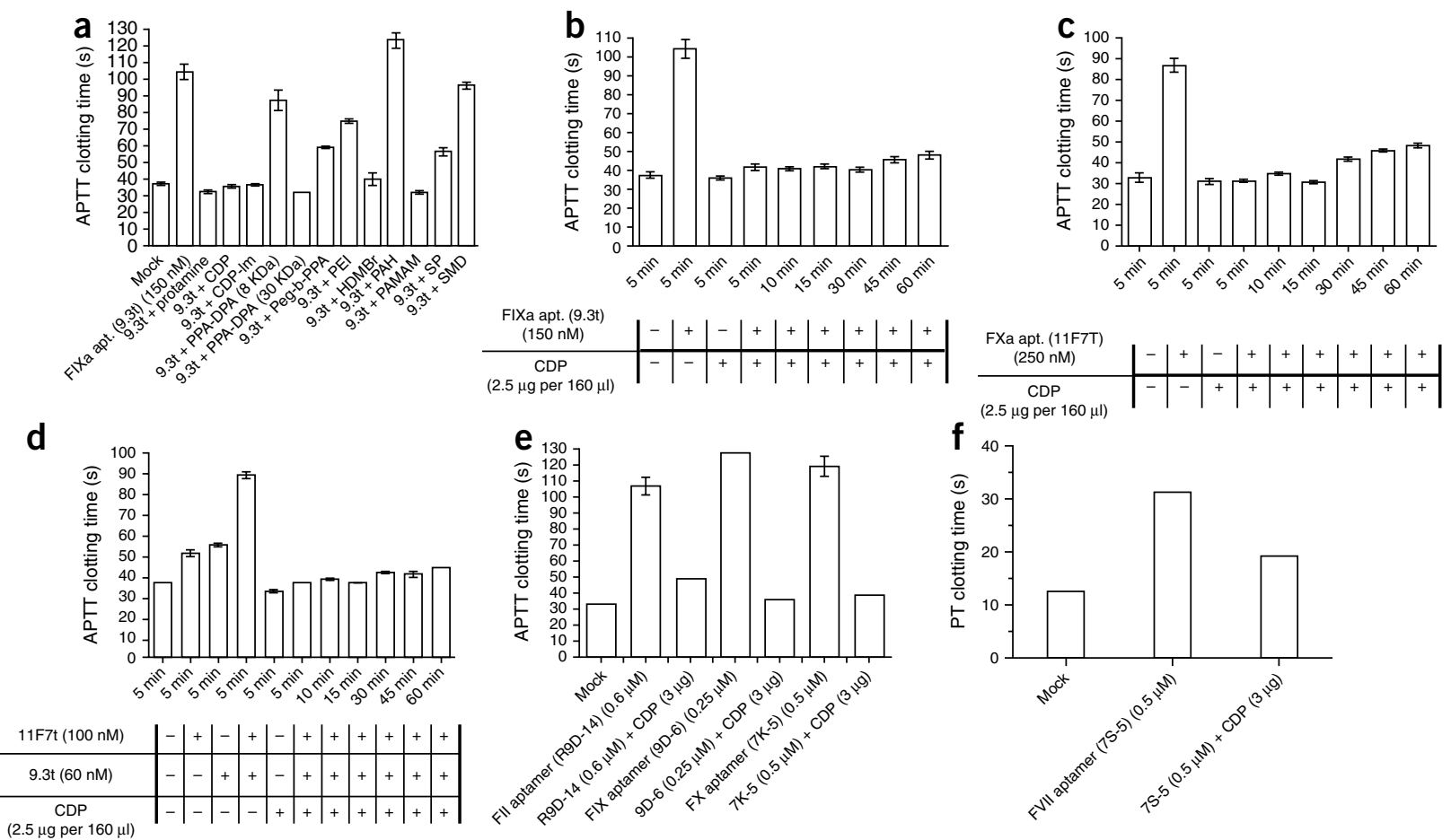

Figure 2 Polymer-mediated reversal of anticoagulant aptamer function. (a) APTT clotting time of normal human plasma in the presence of aptamer 9.3t (150 nM), with and without the addition of protamine or 11 different polymers $(2.5 \mu \mathrm{g}$ per $160 \mu \mathrm{l})$. PEI, polyethyleneimine; PAH, poly(allylamine hydrochloride); $\mathrm{HDMBr}$, hexadimethrine bromide; SP, spermine; SMD, spermidine. (b-e) APTT clotting time of normal human plasma in the presence of aptamer 9.3t (150 nM) (b), aptamer 11F7T (c), aptamers 9.3t and 11F7 (d), aptamers R9D-14, 9D-6 and 7K-5 (e), with and without the addition of CDP. (f) Prothrombin time (PT) clotting time of normal human plasma in the presense of aptamer 7S-1 with and without the addition of CDP. The data are plotted as the means \pm s.e.m. for three independent measurements.

(PPA-DPA) and PAMAM showed considerable and similar affinities for aptamer 9.3t, whereas the binding constants of polybrene and spermine were orders of magnitude lower (Supplementary Table 2). Thus, there is a direct correlation between a polymer's affinity for the aptamer and its potency as an antidote.

Because CDP has high binding affinity for the aptamer and is known to have low toxicity, we tested this polymer in time-course experiments ${ }^{27}$. Similarly to protamine, CDP could rapidly and durably reverse the activity of these two distinct anticoagulant aptamers (9.3t and 11F7T) in vitro (Fig. 2b-d). Subsequently, we examined CDP's ability to reverse the activity of four additional aptamers that target FII, FIX, FX and FVII. CDP could rapidly reverse the activity of each of these aptamers (Fig. 2e,f).

Next, we tested CDP and PPA-DPA for their ability to neutralize the antiplatelet effects of VWF aptamer 9.3 and vWF aptamer 9.14 (Fig. 1a) in a platelet function assay (PFA-100) ${ }^{17}$. vWF aptamers 9.3 and 9.14 have no sequence or structure similarity to the previously tested aptamers, and both could inhibit platelet function in whole blood (Fig. 3a,b).

Figure 3 Polymer-mediated reversal of antiplatelet aptamer function. (a,b) PFA-100 closing time of normal whole blood in the presence of vWF aptamer 9.3 with and without the addition of CDP (a) or PPA-DPA (30 $\mathrm{kDa}$ ) (b). (c) PFA closing time of normal whole blood in the presence of VWF aptamer 9.14 with and without the addition of CDP. The data are plotted as the means \pm s.e.m. for three independent measurements.
Addition of either CDP or PPA-DPA resulted in rapid reversal of vWF aptamer 9.3 antiplatelet activity, with CDP achieving complete reversal at an order of magnitude lower amount than PPA-DPA (Fig. 3a,b). Moreover, CDP was able to rapidly reverse the activity of vWF aptamer 9.14 at this same concentration (Fig. 3c). These experiments further demonstrate that CDP and PPA-DPA can act as sequence-independent antidotes for aptamers. Moreover, these results point to the broad applicability of this approach, as the antidotes work in both plasma and whole blood against eight different aptamers.

Next, we sought to determine whether such universal antidotes are able to reverse aptamer activity in vivo. Results from in vitro experiments (gel electrophoresis and dynamic light scattering) using the same concentrations as anticipated for use in mice showed that CDP is able to bind the aptamer and form a composite entity (Supplementary Fig. 1), and we observed that CDP-Im formed a complex with siRNA when sequentially injected into mice (Supplementary Fig. 2). Therefore, we
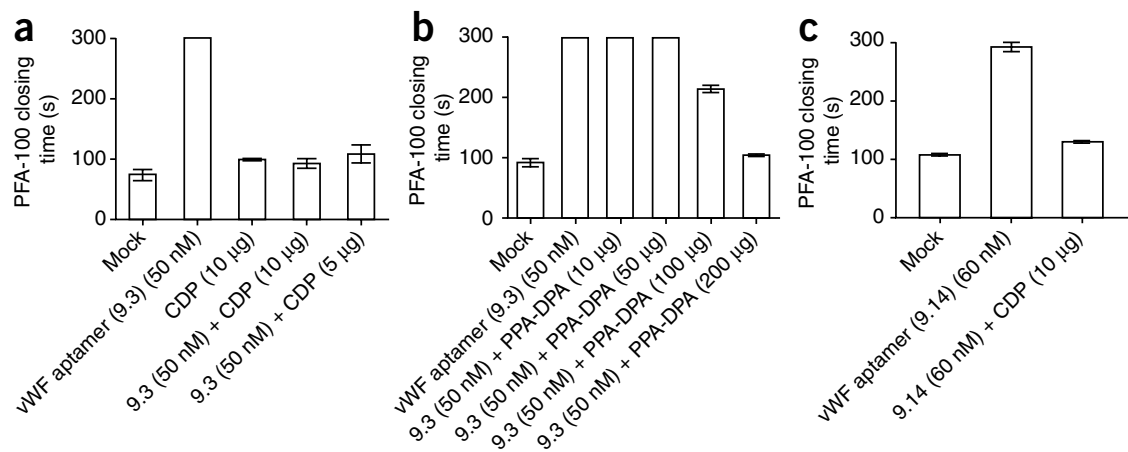

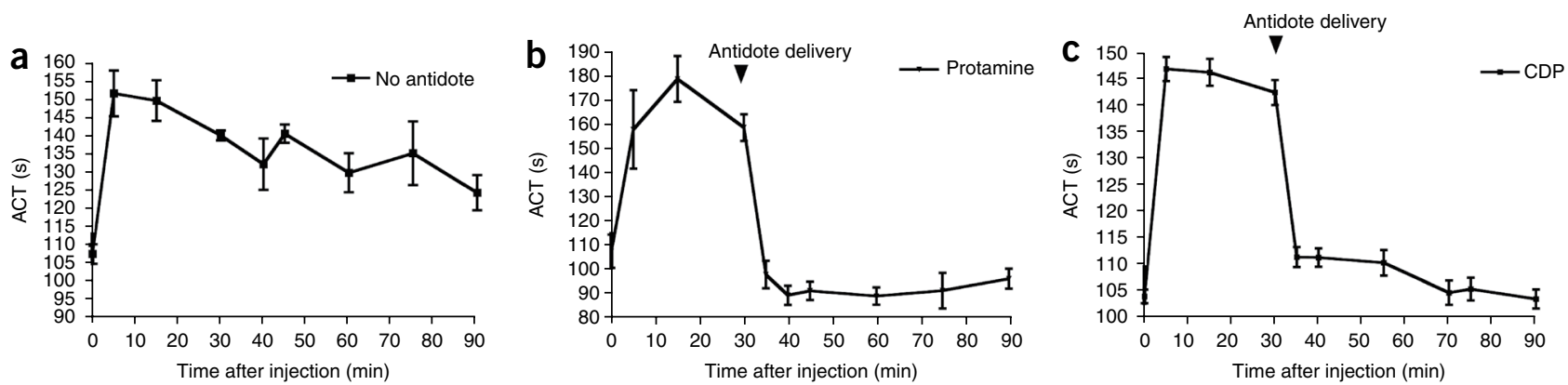

Figure 4 In vivo aptamer and antidote activity. (a-c) ACT clotting times of blood from swine treated with the cholesterol-modified FIXa aptamer 9.3t (Ch-9.3t) in pigs $(n=5)$ alone (a) or with the addition of protamine (b) or CDP (c). The data are plotted as the means \pm s.e.m. for duplicate measurements from each pig.

evaluated the activity of the universal antidotes in a swine anticoagulation model. Pigs $(n=5)$ were anticoagulated with the FIXa aptamer (Ch-9.3t) (0.5 mg per kg body weight) that had been modified with a cholesterol at its $5^{\prime}$ end to improve its circulating half-life ${ }^{19}$ (Fig. 4a). We observed an immediate increase in the activated clotting time (ACT) (from $105 \pm 5 \mathrm{~s}$ to $150 \pm 5 \mathrm{~s}$ ) for the treated pigs. When no antidote was administered, the level of anticoagulation only gradually decreased over the 90-min time frame of the experiment (Fig. 4a). However, administration of protamine (10 mg per kg body weight) resulted in a total reversal of the anticoagulant effect within $5 \mathrm{~min}(n=5)$ (Fig. 4b). In addition, this reversal was sustained for the remainder of the experiment, 60 min (Fig. 4b). Similarly, CDP $(n=5)(2.5 \mathrm{mg}$ per $\mathrm{kg}$ body weight) was also able to rapidly and durably reverse the activity of this aptamer in vivo (Fig. 4c). Furthermore, we did not observe any toxicities after administration of these antidotes during the experiment (Supplementary Fig. 3). All vital signs stayed within error of their baseline levels, with the exception that protamine induced a mild hypotension and CDP a mild hypertension $(<15 \%$ change; Supplementary Fig. 3d,e). These results indicate that both protamine and CDP can act as antidotes for aptamers in vivo.

\section{DISCUSSION}

Between 1998 and 2005, the number of serious adverse drugs events reported to the US Food and Drug Administration increased 2.6-fold, and fatal adverse events increased 2.7-fold to 15,107 events in 2005 (refs. 28,29). Therefore, there is a pressing medical need to develop safer and more controllable therapeutic strategies. Unfortunately, it has been both technically challenging and very expensive to develop antidote molecules to counteract the side effects of most medicines. We have shown that characteristics unique to oligonucleotides can be used to design universal antidotes that can sequester aptamers and reverse their activity, regardless of the aptamer's primary sequence and folded structure. Our initial studies showed that protamine, a commonly used and inexpensive heparin reversal agent with wellknown side effects, can be used as an antidote for multiple aptamers $^{30,31}$. Furthermore, the observation that protamine can neutralize aptamer activity indicates that protamine should be used with caution in patients being treated with oligonucleotide-based drugs, as protamine may unintentionally reverse their activity.

To find universal antidotes with more favorable characteristics such as low toxicity, we tested a number of polymeric gene carriers for their ability to reverse the activity of aptamers. The field of nonviral gene therapy has stimulated the synthesis of many polymers for the delivery of plasmid DNA and siRNA. The rigidity, hydrophobicity and hydrophilicity, charge density, biodegradability and molecular weight of the polymer chain are all parameters that can be adjusted to achieve an optimal complexation with oligonucleotides ${ }^{32,33}$. Moreover, it has previously been shown that a small cationic porphyrin can act as an antidote to a G-quartet-containing thrombin aptamer ${ }^{34}$. Therefore, we screened a number of DNA or siRNA delivery polymers and found that several of them can reverse the activity of multiple aptamers in vitro, and that CDP can also rapidly reverse the activity of an anticoagulant aptamer in pigs.

Previously, we developed a strategy to reverse the activity of aptamers using Watson-Crick base-pairing rules to create a customized antidote oligonucleotide for each aptamer ${ }^{16,19}$. This customization is very costly, because for each aptamer a new antidote oligonucleotide has to be developed, tested and manufactured. Another concern with this approach is that when antidote oligonucleotides bind to aptamers, a double-stranded RNA is formed that may stimulate the innate immune system ${ }^{20,35}$. Although both the RNA aptamer and the antidote oligonucleotide are often comprised of modified oligonucleotides (that is, 2-F and 2-OMe RNA), there is still a danger that these molecules may activate Toll-like receptor 3, as short 2'Ome siRNA duplexes have been reported to induce such effects ${ }^{20}$. Finally, because most indications will not require an antidote for the reversal of drug action $100 \%$ of the time, the added cost of developing a customized antidote is difficult to justify for most drugs that usually are safe but are associated with relatively rare but serious side effects. Thus, we believe that the universal antidote approach will be more broadly applicable than the customized antidote oligonucleotide approach we have described. As with any new therapeutic agent, the safety of the universal antidote molecules will have to be tested in the clinic. Recent studies evaluating the toxicity of CDP-Im in nonhuman primates have shown that such compounds have a favorable safety profile ${ }^{36}$. Thus, we are optimistic that the universal antidote strategy can be rapidly translated into the clinic and believe that safer therapeutic agents may be forthcoming.

\section{METHODS}

Methods and any associated references are available in the online version of the paper at http://www.nature.com/naturemedicine/.

Note: Supplementary information is available on the Nature Medicine website.

\section{ACKNOWLEDGMENTS}

We thank S.M. Nimjee and J. Layzer for helpful discussions and Calando Pharmaceuticals for providing the CDP and CDP-Im polymers. This work was supported by a grant from the US National Institutes of Health (HL065222 to B.A.S.), a predoctoral fellowship from the American Heart Association (0615443U to S.O.) and a grant from the US National Cancer Institute (CA 119347 to M.E.D). 


\section{TECHNICAL REPORTS}

\section{AUTHOR CONTRIBUTIONS}

S.O. designed and performed research, analyzed data and wrote the manuscript; R.T.S.L designed and performed research and analyzed data; K.M.B. performed research; C.M.B. performed research and analyzed data; G.Q. performed research; J.D.H. designed and performed research, provided useful reagents, analyzed data and provided useful discussions; J.Y.-C.L. performed research; B.C.M. performed research; M.E.D. provided useful reagents and discussions; K.W.L. provided useful reagents and discussions; B.A.S. suggested the universal antidote idea, designed and coordinated research, analyzed data and wrote the manuscript.

\section{COMPETING INTERESTS STATEMENT}

The authors declare competing financial interests: details accompany the full-text HTML version of the paper at http://www.nature.com/naturemedicine/.

Published online at http://www.nature.com/naturemedicine/.

Reprints and permissions information is available online at http://npg.nature.com/ reprintsandpermissions/.

1. Dyke, C.K. et al. First-in-human experience of an antidote-controlled anticoagulant using RNA aptamer technology: a phase 1a pharmacodynamic evaluation of a drugantidote pair for the controlled regulation of factor IXa activity. Circulation 114, 2490-2497 (2006).

2. EyetechStudyGroup. Preclinical and phase $1 \mathrm{~A}$ clinical evaluation of an anti-VEGF pegylated aptamer (EYEO01) for the treatment of exudative age-related macular degeneration. Retina 22, 143-152 (2002).

3. EyetechStudyGroup. Anti-vascular endothelial growth factor therapy for subfoveal choroidal neovascularization secondary to age-related macular degeneration: phase II study results. Ophthalmology 110, 979-986 (2003).

4. Gilbert, J.C. et al. First-in-human evaluation of anti von Willebrand factor therapeutic aptamer ARC1779 in healthy volunteers. Circulation 116, 2678-2686 (2007).

5. Lee, J.H. et al. A therapeutic aptamer inhibits angiogenesis by specifically targeting the heparin binding domain of VEGF165. Proc. Natl. Acad. Sci. USA 102, 1890218907 (2005)

6. McNamara, J.O. et al. Multivalent 4-1BB binding aptamers costimulate CD8+ T cells and inhibit tumor growth in mice. J. Clin. Invest. 118, 376-386 (2008).

7. Nimjee, S.M., Rusconi, C.P. \& Sullenger, B.A. Aptamers: an emerging class of therapeutics. Annu. Rev. Med. 56, 555-583 (2005).

8. Sullenger, B.A., Gallardo, H.F., Ungers, G.E. \& Gilboa, E. Overexpression of TAR sequences renders cells resistant to human immunodeficiency virus replication. Cell 63, 601-608 (1990)

9. Anderson, J. et al. Safety and efficacy of a lentiviral vector containing three anti-HIV genes-CCR5 ribozyme, tat-rev siRNA, and TAR decoy-in SCID-hu mouse-derived T cells. Mol. Ther. 15, 1182-1188 (2007).

10. Ellington, A.D. \& Szostak, J.W. In vitro selection of RNA molecules that bind specific ligands. Nature 346, 818-822 (1990).

11. Tuerk, C. \& Gold, L. Systematic evolution of ligands by exponential enrichment: RNA ligands to bacteriophage T4 DNA polymerase. Science 249, 505-510 (1990).

12. Becker, R.C., Rusconi, C. \& Sullenger, B. Nucleic acid aptamers in therapeutic anticoagulation. Technology, development and clinical application. Thromb. Haemost. 93, 1014-1020 (2005).

13. Nimjee, S.M., Rusconi, C.P., Harrington, R.A. \& Sullenger, B.A. The potential of aptamers as anticoagulants. Trends Cardiovasc. Med. 15, 41-45 (2005).
14. Fanikos, J. et al. Adverse drug events in hospitalized cardiac patients. Am. J. Cardiol. 100, 1465-1469 (2007).

15. Nimjee, S.M. et al. A novel antidote-controlled anticoagulant reduces thrombin generation and inflammation and improves cardiac function in cardiopulmonary bypass surgery. Mol. Ther. 14, 408-415 (2006).

16. Rusconi, C.P. et al. RNA aptamers as reversible antagonists of coagulation factor IXa. Nature 419, 90-94 (2002).

17. Oney, S. et al. Antidote-controlled platelet inhibition targeting von Willebrand factor with aptamers. Oligonucleotides 17, 265-274 (2007)

18. Rusconi, C.P., Yeh, A., Lyerly, H.K., Lawson, J.H. \& Sullenger, B.A. Blocking the initiation of coagulation by RNA aptamers to factor VIla. Thromb. Haemost. 84 841-848 (2000).

19. Rusconi, C.P. et al. Antidote-mediated control of an anticoagulant aptamer in vivo. Nat. Biotechnol. 22, 1423-1428 (2004).

20. Kleinman, M.E. et al. Sequence- and target-independent angiogenesis suppression by siRNA via TLR3. Nature 452, 591-597 (2008).

21. White, R. et al. Generation of species cross-reactive aptamers using "toggle" SELEX Mol. Ther. 4, 567-573 (2001)

22. Zuker, M. Mfold web server for nucleic acid folding and hybridization prediction Nucleic Acids Res. 31, 3406-3415 (2003).

23. Hird, R.B. et al. Direct effects of protamine sulfate on myocyte contractile processes Cellular and molecular mechanisms. Circulation 92, II433-II446 (1995).

24. Porsche, R. \& Brenner, Z.R. Allergy to protamine sulfate. Heart Lung 28, 418-428 (1999).

25. Shigeta, O. et al. Low-dose protamine based on heparin-protamine titration method reduces platelet dysfunction after cardiopulmonary bypass. J. Thorac. Cardiovasc. Surg. 118, 354-360 (1999).

26. Welsby, I.J. et al. Hemodynamic changes after protamine administration: association with mortality after coronary artery bypass surgery. Anesthesiology 102, 308-314 (2005).

27. Gonzalez, H., Hwang, S.J. \& Davis, M.E. New class of polymers for the delivery of macromolecular therapeutics. Bioconjug. Chem. 10, 1068-1074 (1999).

28. Moore, T.J., Cohen, M.R. \& Furberg, C.D. Serious adverse drug events reported to the Food and Drug Administration, 1998-2005. Arch. Intern. Med. 167 1752-1759 (2007)

29. Lazarou, J., Pomeranz, B.H. \& Corey, P.N. Incidence of adverse drug reactions in hospitalized patients: a meta-analysis of prospective studies. J. Am. Med. Assoc. 279, 1200-1205 (1998).

30. Carr, J.A. \& Silverman, N. The heparin-protamine interaction. A review. J. Cardiovasc Surg. (Torino) 40, 659-666 (1999).

31. Stanker, L.H., Wyrobek, A., McKeown, C. \& Balhorn, R. Identification of the binding site of two monoclonal antibodies to human protamine. Mol. Immunol. 30, 1633-1638 (1993).

32. Davis, M.E. et al. Self-assembling nucleic acid delivery vehicles via linear, watersoluble, cyclodextrin-containing polymers. Curr. Med. Chem. 11, 179-197 (2004).

33. Mao, H.Q. \& Leong, K.W. Design of polyphosphoester-DNA nanoparticles for nonviral gene delivery. Adv. Genet. 53, 275-306 (2005).

34. Joachimi, A., Mayer, G. \& Hartig, J.S. A new anticoagulant-antidote pair: control of thrombin activity by aptamers and porphyrins. J. Am. Chem. Soc. 129, 3036-3037 (2007)

35. Schröder, M. \& Bowie, A.G. TLR3 in antiviral immunity: key player or bystander? Trends Immunol. 26, 462-468 (2005).

36. Heidel, J.D. et al. Administration in non-human primates of escalating intravenous doses of targeted nanoparticles containing ribonucleotide reductase subunit M2 siRNA. Proc. Natl. Acad. Sci. USA 104, 5715-5721 (2007). 


\section{ONLINE METHODS}

Clotting assays. We performed APTT assays with a model ST4 mechanical coagulometer (Diagnostica Stago Inc.). We incubated pooled normal human plasma $(50 \mu \mathrm{l})$ (George King Biomedical) at $37^{\circ} \mathrm{C}$ for $5 \mathrm{~min}$ followed by the addition of platelin reagent $(50 \mu \mathrm{l})$ (Trinity Biotech) and aptamer (in wash buffer; $40 \mathrm{mM}$ HEPES, pH 7.5, $150 \mathrm{mM} \mathrm{NaCl}$ and $\left.2 \mathrm{mM} \mathrm{CaCl}_{2}\right)(5 \mu \mathrm{l})$ or wash buffer alone and incubation for $5 \mathrm{~min}$ at $37^{\circ} \mathrm{C}$. We then added antidote molecule or buffer $(5 \mu \mathrm{l})$ and incubated at $37^{\circ} \mathrm{C}$ for $5 \mathrm{~min}$ followed by the addition of $25 \mathrm{mM} \mathrm{CaCl} 2(50 \mu \mathrm{l})$ to initiate the clotting reaction ${ }^{16}$. Data are shown as the change in clot time. We performed all reactions in triplicate.

Platelet function assays. The Platelet Function Analyzer, PFA-100 (Dade Behring), measures platelet function in terms of clot formation time. In this assay, we used collagen and ADP cartridges (Dade Behring) to activate the platelets. We mixed $800 \mu \mathrm{l}$ of whole blood with aptamer in platelet binding buffer $(40 \mu \mathrm{l})\left(150 \mathrm{mM} \mathrm{NaCl}, 20 \mathrm{mM}\right.$ HEPES pH 7.4, $5 \mathrm{mM} \mathrm{KCl}, 1 \mathrm{mM} \mathrm{MgCl}_{2}$ and $1 \mathrm{mM} \mathrm{CaCl}_{2}$ ) and incubated for $5 \mathrm{~min}$ at $25^{\circ} \mathrm{C}$. We then added antidote molecule or platelet buffer $(40 \mu \mathrm{l})$ and incubated for $5 \mathrm{~min}$ at $25^{\circ} \mathrm{C}$. We added this mixture to a collagen/ADP cartridge and tested for its closing time ${ }^{37}$. The maximum closing time of the PFA-100 is $300 \mathrm{~s}$. Data are shown as the change in closing time. We performed all reactions in triplicate.

Isothermal calorimetry. We conducted the ITC measurements with a thermostatic and fully computer-operated MCS-ITC calorimeter from MicroCal. We titrated 10- $\mu \mathrm{l}$ aliquots into the calorimetric cell every $5 \mathrm{~min}$ over a 2 -h period at $298 \mathrm{~K}$. We carried out a blank run for each system studied where we titrated the titrant into a cell containing only PBS to allow corrections for the heat effects due to the dilutions to be made. We performed data analysis using the customized ITC module of the Origin 5.0 software package and a least-squares fitting procedure to fit the data to the two-sites binding model.

In vitro assessment of CDP-9.3t interaction by gel electrophoresis and dynamic light scattering. We performed gel electrophoresis by adding a $5-\mathrm{ml}$ volume of $0.01 \mathrm{mg} \mathrm{m}^{-1} 9.3 \mathrm{t}$ in $1 \times \mathrm{PBS}$ and an equal volume of CDP in $1 \times \mathrm{PBS}$. We did this six times in parallel with CDP solutions of various concentrations to give resulting solutions having CDP/9.3t ratios (wt/wt) of 0:1 (lane 2), 2:1 (lane 3), 4:1 (lane 4), 6:1 (lane 5), 8:1 (lane 6) and 10:1 (lane 7). To each of these $10-\mu \mathrm{l}$ solutions, we added $2 \mu \mathrm{l}$ of nucleic acid loading buffer (Bio-Rad), and we loaded $10 \mu \mathrm{l}$ of each resulting solution per well of a $15 \%$ polyacrylamide/TBE gel and electrophoresed $(1 \mathrm{~h}, 100 \mathrm{~V})$. We then incubated the gel in $1 \times \mathrm{TBE}$ buffer containing $0.5 \mathrm{mg} \mathrm{ml}^{-1}$ ethidium bromide for $30 \mathrm{~min}$ and visualized it. We analyzed CDP alone $\left(0.0335 \mathrm{mg} \mathrm{m}^{-1}\right.$ in $\left.1 \times \mathrm{PBS}\right)$, $9.3 \mathrm{t}$ alone $\left(0.005 \mathrm{mg} \mathrm{ml}^{-1}\right.$ in $1 \times$ PBS $)$ and CDP plus $9.3 \mathrm{t}\left(0.0335 \mathrm{mg} \mathrm{m}^{-1} \mathrm{CDP}, 0.005 \mathrm{mg} \mathrm{ml}^{-1} 9.3 \mathrm{t} ; 6.7: 1 \mathrm{wt} / \mathrm{wt}\right.$ in $1 \times$ PBS) by dynamic light scattering using a ZetaPALS particle size analyzer. We performed three consecutive 3-min runs for each sample and determined the effective diameter and average particle count rate.

Analysis of CDP-Im-siRNA complex formation in vivo. We gave four female $\mathrm{BALB} / \mathrm{c}$ mice (Jackson Laboratories) a single tail-vein injection of naked, nonchemically modified siRNA. One minute after this injection, we gave two of the mice a separate tail-vein injection of CDP-Im along with two other typical nanoparticle components, $\mathrm{AD}-\mathrm{PEG}$ and $\mathrm{AD}-\mathrm{PEG}-\mathrm{Tf}^{36}$. Two minutes later, we collected blood from all mice and centrifuged it to separate serum. We then electrophoresed serum samples on a 15\% TBE polyacrylamide gel and visualized the gel with ethidium bromide.

Swine systemic anticoagulation and reversal study. We randomly assigned outbred pigs (2.5-3.5 kg; Wesley Looper Farms) to treatment groups. For all groups, we induced anesthesia by intramuscular injection of ketamine $(22 \mathrm{mg}$ per $\mathrm{kg}$ body weight) and acepromazine (1.1 mg per kg body weight). We then placed a catheter in the ear vein, through which we maintained anesthesia with fentanyl, first with a $100 \mu \mathrm{g}$ per kg body weight bolus and then with a continuous infusion of $60 \mu \mathrm{g}$ per kg body weight per $\mathrm{h}$. We then intubated and mechanically ventilated the pigs. After placement of the esophageal or rectal temperature probe and pulse oximeter monitor, we cannulated the femoral artery and vein. We used the arterial line as a means to continuously monitor mean arterial blood pressure and heart rate, as well as to remove blood samples for evaluation of ACT. After determining baseline ACT values, we used the venous line to administer the FIXa aptamer $(0.5 \mathrm{mg}$ per kg body weight). We then drew blood samples from the arterial line at 5,15 and $30 \mathrm{~min}$ after aptamer administration. We measured the ACT values by using Hemochron Jr. Signature Microcoagulation System (ITC). For experiments involving antidote administration, we gave $40 \mathrm{mg}$ protamine $\left(10 \mathrm{mg} \mathrm{ml}^{-1}\right)$ or $10 \mathrm{mg} \mathrm{CDP}\left(10 \mathrm{mg} \mathrm{ml}^{-1}\right)$ over $5 \mathrm{~min}$ via the femoral vein catheter at $30 \mathrm{~min}$ after aptamer injection. For all pigs, we took subsequent blood samples at 35, 40, 55, 60, 75, and $90 \mathrm{~min}$ after aptamer administration. All data points were done in duplicate per pig. At the closure of the experiment, we killed the pigs with Euthasol (175 mg per kg body weight) via femoral vein.

All animals received humane treatment in accordance with the Guide for the Care and Use of Laboratory Animals published by the National Institutes of Health, as approved by the Duke University Animal Care and Use Committee.

37. Harrison, P. Platelet function analysis. Blood Rev. 19, 111-123 (2005). 